\title{
The role of periostin in kidney diseases
}

\author{
AGNIESZKA TURCZYN, MAEGORZATA PAŃCZYK-TOMASZEWSKA
}

Department of Pediatrics and Nephrology, Medical University of Warsaw, Warsaw, Poland

\begin{abstract}
Chronic kidney disease (CKD) is a multi-symptomatic condition resulting from irreversible functional and structural damage to the kidneys. Therefore, finding a specific and sensitive marker to predict the development and progression of CKD is of great interest. Periostin is a matricellular protein involved in tissue remodeling and wound healing. It is highly expressed in various types of kidney diseases, especially in conditions associated with progressive renal fibrosis, while its expression in healthy kidneys is not significant. Numerous experimental and human adult studies indicate the role of periostin in the pathogenesis of various types of kidney disease, though the mechanism of action of periostin appears to be diverse and varies depending on the conditions. The article summarizes current knowledge on the possible roles of periostin in the pathogenesis of kidney injury and its position as a marker in various human renal pathologies. The studies performed so far indicate the potential of urinary and tissue periostin as a promising biomarker of CKD progression.
\end{abstract}

Key words: fibrosis, kidney, periostin, inflammation.

(Cent Eur J Immunol 2021; 46 (4): 494-501)

\section{Introduction}

According to Kidney Disease: Improving Global Outcome (KDIGO) 2012 recommendations, chronic kidney disease (CKD) is defined as abnormalities of kidney structure or function, present for more than three months, with serious implications for health. Chronic kidney disease is classified based on cause, glomerular filtration rate (GFR) category, and albuminuria category (CGA - cause, GFR, albuminuria classification) [1]. The incidence of CKD in children is estimated at 7.7-12.1 and prevalence at 59-74.7 cases per million children [2,3]. Congenital anomalies of the kidney and urinary tract are the leading cause of CKD in the pediatric population [3].

Chronic kidney disease is a multi-symptomatic condition resulting from irreversible functional and structural damage to the kidneys. Renal fibrosis is the key process responsible for the initiation and progression of CKD, leading ultimately to end-stage renal disease (ESRD). Renal fibrosis is a process characterized by excessive deposition of extracellular matrix proteins (ECM) within the kidney interstitium, leading to interstitial scarring, glomerular sclerosis, tubular and vascular atrophy [4-6]. Since CKD places a significant burden on public health institutions, finding a specific and sensitive marker to predict the development and progression of CKD is of great interest.

Extracellular matrix proteins are highly specialized molecules that form a scaffold for various cell types. They bind to numerous cell surface receptors and regulate various matrix processes. Apart from their continuous role in remodeling, ECM proteins are important for wound healing, inflammation, fibrosis, and cancer progression. The components of the extracellular matrix include collagens, elastin, fibronectin, thrombospondins (TSPs), osteopontin, secreted cysteine-rich acid protein (SPARC), and periostin [7].

Both experimental [8] and human adult [9] studies suggest that periostin is involved in the progression of renal injury and could be used as an early marker reflecting CKD progression. The aim of this manuscript is to summarize the current knowledge about the role of periostin in the progression of CKD and its role as a potent biomarker in various kidney pathologies.

\section{The structure and expression of periostin}

Periostin was first described in 1993 as an osteoblast-specific factor 2 (Osf-2) [10]. The periostin gene consists of 23 exons and is located on the 13q13.3 chromosome. Periostin is a protein with a molecular weight of $90 \mathrm{kDa}$. It belongs to the fasciclin family, together with transforming growth factor $\beta$-induced protein (TGFBIp, Big-H3), which shows $48 \%$ homology with periostin $[11,12]$. Periostin is composed of an N-terminal secretory signal peptide, a small EMI domain, four repetitive and conserved cysteine-rich fasciclin (FAS1) domains, and a carboxyl-terminal domain (CTD) [11, 13, 14]. 
The EMI domain is responsible for the multimerization of the protein, protein-to-protein interactions, and heparin binding. Through the EMI domain, periostin directly interacts with the ECM proteins: collagen I, fibronectin, and Notch1 and is involved in collagen fibrillogenesis. It forms a scaffold for ECM proteins and increases the secretion of fibronectin into the extracellular matrix [15-17].

FAS1 domains of periostin consist of 150 amino acids, of which ten are conserved. FAS1 domains serve as a ligand for integrin $\alpha v \beta 1, \alpha v \beta 3, \alpha v \beta 5, \alpha 6 \beta 4$ receptors and for the ECM protein tenascin-C. Integrins promote cell adhesion, proliferation, and migration, as well as the epithelial-mesenchymal transition (EMT) [12, 15, 16, 18-20]. $\mathrm{N}$-terminal secretory signal peptide recognizes vitamin Kdependent $\gamma$-glutamyl carboxylase, which catalyzes the conversion of glutamic acid to $\gamma$-carboxyglutamate (Gla). Gla residues bind calcium ions and may play a role in tissue calcification [21]. However, it is uncertain whether periostin promotes or prevents the calcification process [22].

The amino acid sequence of CTD varies, as it is alternatively spliced between 17 and 21 exons with consequent formation of shorter forms of the protein [13]. The number and the type of periostin isoforms vary depending on tissue or organ. Similar variability also occurs during embryogenesis, including fetal kidneys and lungs [23-25]. So far, eight shorter variants of periostin have been described [23, 26]. The major factor inducing alternative periosteal splicing is TGF- $\beta$ [13], and the role of the shorter forms of periostin is not fully understood and requires further research.

Expression of the periostin gene is under the control of numerous cytokines and hormones. The regulators of periostin expression include: TGF- $\beta 1$, TGF- $\beta 2$, TGF- $\beta 3$, basic helix-loop-helix (bHLH) transcription factor, bone morphogenetic protein-2 (BMP-2) and BMP-4, platelet-derived growth factor (PDGF), vascular endothelial factor growth factor (VEGF), connective tissue growth factor-2 (CTGF-2), angiotensin II, interleukin (IL)-3, IL-4, IL-6, and IL-13 [19, 27] (Fig. 1).

Periostin is expressed predominantly in areas rich in connective tissue with the highest content in ligaments, aorta, lower digestive tract, placenta, uterus, thyroid gland, and breasts $[13,28]$. It is important for maintaining the integrity of the periodontal ligament, and its high expression enables the teeth to be fixed to the bone $[14,29,30]$.

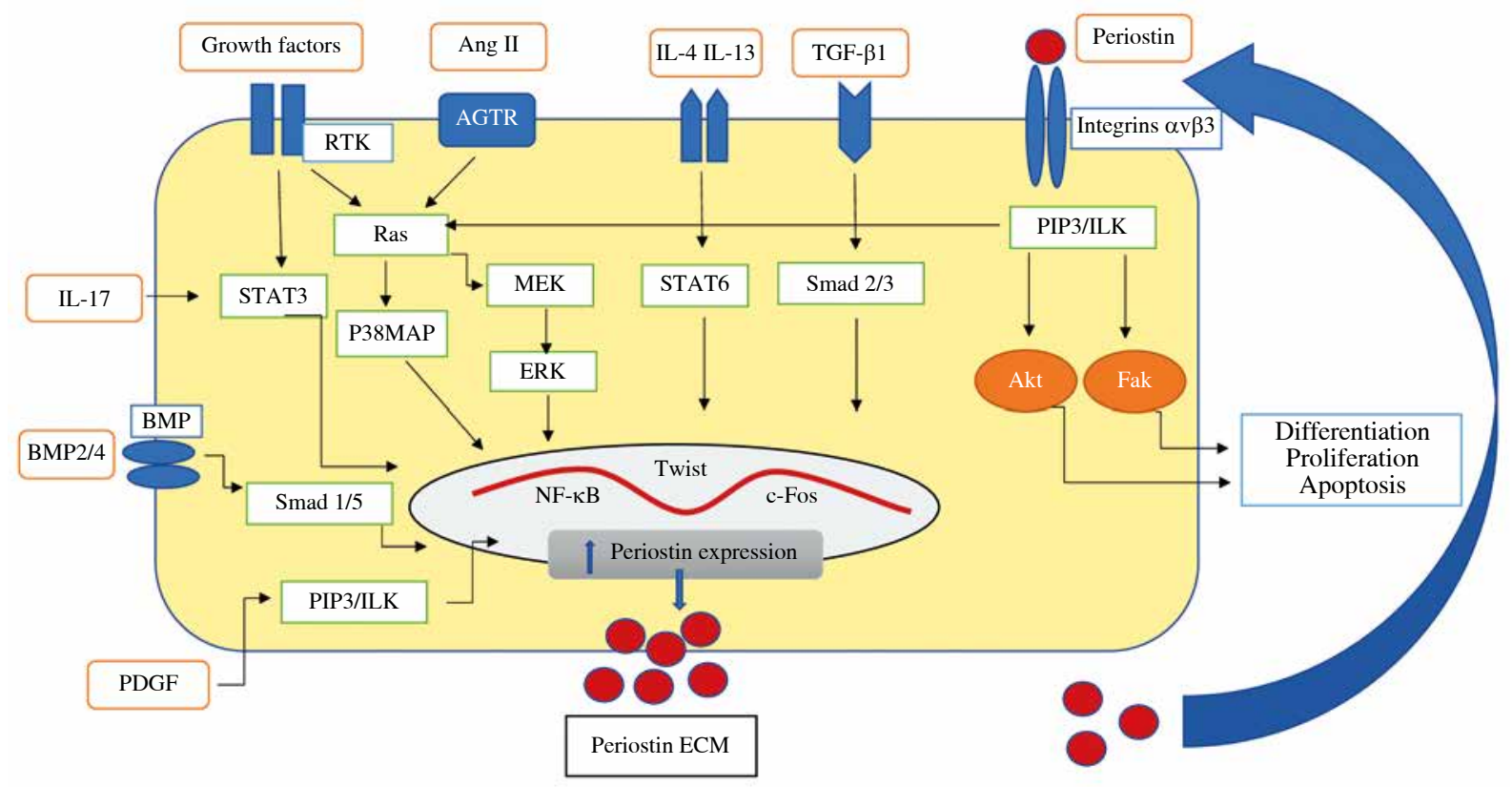

Fig. 1. The diagram shows the factors that induce periostin secretion in the kidney. At first, as a consequence of mechanical stress or inflammation, transforming growth factor $\beta$ (TGF- $\beta$ ) and/or IL-4, IL-13 are released. These cytokines triggered overexpression of periostin through transcription factors such as nuclear factor-kappa $\mathrm{B}(\mathrm{NF}-\kappa \mathrm{B})$, Twist-related protein (Twist), c-Fos, signal transducer, and activator of transcription 1 (STAT1) signal transducer and activator of transcription 6 (STAT6), c-Jun. Then periostin is secreted and accumulated outside cells within ECM, where it binds to integrin receptor, induces integrin-linked kinase (ILK), and activates signaling pathways involved in inflammation and fibrosis in the kidneys. Also, periostin's ability to activate latent TGF- $\beta$ in the feedback loop has been observed. Other factors and signaling pathways inducing periostin expression are proposed: Ang II, PDGF, IL-17, BMP-2, BMP-4; p38 mitogen-activated protein kinases (p38MAPK), extracellular signal-regulated kinase (ERK), mitogen-activated protein kinase (MEK), phosphatidylinositol-3 kinase (PIP3), receptor tyrosine kinases (RTK), focal adhesion kinase (FAK), serine/threonine-protein kinase (Akt); signal transducer and activator of transcription (STAT 3), angiotensin II receptor (AGTR) 
It plays a role in bone tissue remodeling, repair, and homeostasis [14, 19, 31, 32]. Numerous studies confirm the role of periostin in wound healing and scar formation. Increased periostin expression has been reported in various autoimmune entities such as psoriasis and scleroderma [33-36]. Periostin plays a role in the differentiation of the heart vessels, development of valves, and formation of the fibrous skeleton of the heart [37-40]. Its involvement in the pathogenesis of arterial hypertension, progression of atherosclerosis, and restenosis after a percutaneous coronary intervention has been described. It also promotes the progression of degenerative heart disease and its fibrosis $[39,41,42]$.

Increased expression of periostin has been reported in the course of numerous allergic and lung pathologies, e.g. asthma, pulmonary fibrosis, rhinosinusitis, and nasal polyps [43-47]. In the neoplastic process, periostin plays an important role in angiogenesis, cell migration, and the formation of metastases. Increased periostin expression has been found in many types of cancer, including thymoma, lung, breast, pancreatic, bladder, and kidney cancer $[19,23,48-50]$.

\section{Periostin expression in the kidney}

The analysis of kidney biopsies from healthy kidney donors showed a constant presence of periostin in the vascular pole of the glomerulus and around Bowman's capsule. Periostin was not present in tubules [51]. Of note, some other studies showed that periostin expression was not revealed in kidney specimens from people without renal pathology $[9,52,53]$. Inversely, periostin was detected during nephrogenesis in the kidney mesenchyme, developing nephrons, ureteral epithelium, and kidney vessels [54].

Numerous experimental models revealed substantially increased periostin expression in various kidney pathology conditions, especially in progressive renal fibrosis. Experimental studies have shown upregulation of periostin at the RNA and protein level in many CKD models, including the 5/6 nephrectomy model, streptozotocin-induced diabetic nephropathy, and unilateral ureteral obstruction (UUO). Periostin overexpression was also observed in the kidneys of aging mice. Furthermore, its expression increased significantly with the duration of CKD $[55,56]$. For example, in UUO, periostin was initially present in the collecting tubules (the first to be damaged in obstructive nephropathy) and next in the distal and proximal tubules, which is consistent with disease progression [56].

In line with the results of experimental studies, a significant increase in the expression of periostin in renal tissue during progressive renal damage was observed in adult humans [51, 52]; e.g., in autosomal dominant polycystic kidney disease, periostin is expressed in the epithelial cells of the cyst, cyst extracellular matrix and in the fluid [57].
The location of periostin depending on the disease entity is shown in Table 1.

\section{Periostin function in the kidneys}

The exact role of periostin in the kidney in health and disease is yet to be uncovered. Probably, periostin is involved in normal nephrogenesis. During kidney development, periostin is highly expressed in the renal interstitium and may play a role in tubulogenesis and vasculogenesis. Once kidney embryogenesis is completed, its expression significantly decreases [58]. Most likely other ECM proteins replace periostin function in later stages of nephrogenesis [54]. While the role of periostin in healthy kidney tissue appears to be small, this protein may be crucial in inflammation and fibrosis of the kidney.

In the experimental CKD models, including UUO and streptozotocin-induced diabetic nephropathy, periostin mRNA and protein were substantially increased, mainly in the cytoplasm of tubules [55]. The major sources of periostin in tubular interstitium are fibroblasts and myofibroblasts [51]. Due to the strong secretory signal, periostin appears to be rapidly secreted from cells, where it accumulates within the matrix [16]. Along with these effects, difficulties in detecting it in cytoplasm or body fluids could be observed [59]. As a modulator of cell-matrix interaction, periostin promotes collagen cross-linking by supporting BMP-1 mediated activation of matricellular lysyl oxidase (LOX). Studies demonstrated the involvement of LOX in kidney fibrosis [60].

Numerous mechanisms of periostin action in kidney pathology have been proposed.

Firstly, as the periostin FAS1 domain binds to integrin receptors, periostin can activate integrin-linked kinase (ILK), thus influencing proliferation and apoptosis of renal cells as revealed in the experimental mouse model of glomerulonephritis [61]. The periostin-ILK signal pathway has been found to participate also in renal cyst growth [57]. In addition, periostin has been shown to strongly induce the expression of integrin $\beta 3$, which activates the inflammatory process [62].

Excessive activation of the renin-angiotensin-aldosterone system (RAAS) is a key mechanism leading to the progression of renal fibrosis. Activation of RAAS could be a link between periostin and renal damage. Increased periostin levels in fibroblasts and vascular smooth muscle cells in rats in response to chronic infusion of angiotensin II have been revealed [63]. Another paper pointed out that periostin could contribute to oxidative stress and was upregulated by angiotensin II via the reactive oxygen species signaling pathway in fibroblasts of hypertensive rats [64]. The mutual relation between RAAS and periostin may be more complex as periostin downregulation attenuated 5/6 nephrectomy-induced intrarenal RAAS activation and renal tissue fibrosis [65]. In a mouse model of hyperten- 
Table 1. Localization of periostin in kidneys

\begin{tabular}{lcccc}
\hline Author & Year & Disease & $\begin{array}{c}\text { Object } \\
\text { of the study }\end{array}$ & Periostin localization \\
\hline Wallace et al. [57] & 2008 & ADPKD & Human & Epithelial cells of the cyst, cyst extracellular matrix, cyst fluid \\
\hline Sen et al. [51] & 2011 & $\begin{array}{c}\text { Kidneys removed } \\
\text { for transplantation }\end{array}$ & Human & $\begin{array}{c}\text { Vascular pole of the kidney and glomerulus, areas of } \\
\text { interstitial fibrosis: tunica media of the renal arteries, tubular } \\
\text { cells in the area of fibrosis and inflammatory infiltrations, } \\
\text { extracellular matrix, and area of tubular atrophy; upregulated } \\
\text { in the interstitium and glomerulus, exacerbated with renal } \\
\text { function deterionation }\end{array}$ \\
\hline
\end{tabular}

\begin{tabular}{lcccc}
\hline Sen et al. [51] & 2011 & $\begin{array}{c}\text { MGN, lupus } \\
\text { nephropathy }\end{array}$ & Human & Mesangial proliferation, glomerular vascular area \\
\hline Sen et al. [51] & 2011 & FSGS & Human & $\begin{array}{c}\text { Glomerular sclerosis area, mesangium area as in healthy } \\
\text { people }\end{array}$ \\
\hline Sen et al. [51] & 2011 & IgAN & Human & No significant overexpression observed \\
\hline Sen et al. [51] & 2011 & $\begin{array}{c}\text { Healthy transplant } \\
\text { donors }\end{array}$ & Human & $\begin{array}{c}\text { Glomerulus in the area of the vascular pole and Bowman's } \\
\text { capsule, no expression in tubular cells }\end{array}$ \\
\hline Sorocos et al. [54] & 2011 & $\begin{array}{c}\text { Kidney and ureter } \\
\text { development }\end{array}$ & Mice & $\begin{array}{c}\text { Mesenchyme surrounding the kidney and the ureter, renal } \\
\text { stroma, metanephric mesenchyme, ureter epithelium and } \\
\text { developing nephrons, renal and intrarenal artery smooth } \\
\text { muscle cells, ureteral smooth muscle cells }\end{array}$ \\
\hline
\end{tabular}

\begin{tabular}{|c|c|c|c|c|}
\hline Satirapoj et al. [55] & 2012 & $\begin{array}{l}5 / 6 \mathrm{Nx} \\
\text { UUO } \\
\text { SZ-DN DBA2J } \\
\text { aged DBA2J }\end{array}$ & $\begin{array}{l}\text { Rat } \\
\text { mice }\end{array}$ & $\begin{array}{l}\text { Areas of necrosis, infarction, apical part of tubular cytoplasm } \\
\text { (gradual increase in tubular expression along with CKD } \\
\text { duration), tubular cells exfoliating into the lumen, area around } \\
\text { renal arteries and arterioles, extracellular matrix } \\
\text { of sclerotic glomeruli }\end{array}$ \\
\hline Guerrot et al. [8] & 2012 & $\begin{array}{l}\text { Hypertensive } \\
\text { nephropathy }\end{array}$ & Rat & $\begin{array}{l}\text { Intima and media, de novo interstitium, proximity to the most } \\
\text { advanced lesions in the glomerular and interstitial vessels } \\
\text { Normal rat kidney - low expression in the area of the median } \\
\text { membrane of the renal arteries and arterioles }\end{array}$ \\
\hline Mael-Ainin et al. [56] & 2014 & UUO & Mice & $\begin{array}{l}\text { Renal tubules, initially collecting tubules, next distal } \\
\text { and proximal tubules }\end{array}$ \\
\hline Satirapoj et al. [53] & 2014 & CAN & Human & $\begin{array}{l}\text { Sclerotic glomerulus, peri-glomerular and interstitial areas } \\
\text { around renal arteries and arterioles, tubular cytoplasm in the } \\
\text { area of inflammatory infiltrate in the interstitium and fibrosis } \\
\text { Control group: no significant expression }\end{array}$ \\
\hline Satirapoj et al. [9] & 2015 & Type 2 diabetes & Human & $\begin{array}{l}\text { Glomerular sclerosis, the area of periglobular fibrosis, } \\
\text { Bowman's capsule, ischemic lesions in the glomerulus, } \\
\text { cytoplasm of atrophic and non-atrophic tubules } \\
\text { Control group: no expression }\end{array}$ \\
\hline
\end{tabular}

Wantanasiri et al. [52] 2015 Lupus nephropathy Human Periglomerular, sclerotic glomeruli, interstitial fibrosis,
fibrotic vessels, renal tubular epithelial cells, atrophic tubules, exfoliated tubular cells

Normal kidneys: no expression

\begin{tabular}{lcccc}
\hline Zhao et al. [77] & 2017 & Lupus nephropathy & Mice & Tubular cells, glomerular mesangium \\
\hline An et al. [74] & 2018 & Unilateral I/R model & Mice & Tubular cells \\
\hline Alferi et al. [78] & 2019 & $\begin{array}{c}\text { Kidney removed } \\
\text { for transplant }\end{array}$ & Human & $\begin{array}{c}\text { Interstitial and glomerular area with intermediate } \\
\text { and advanced lesions }\end{array}$ \\
\hline Kormann et al. [75] & 2020 & I/R model & Mice & $\begin{array}{c}\text { The area of interstitial fibrosis, damaged proximal tubules } \\
\text { and Henle's loop, area of distal and collecting tubules }\end{array}$ \\
\hline
\end{tabular}

5/6 Nx-5/6 nephrectomy (Nx), UOO - unilateral ureteral obstruction, SZ-DN DBA2J mice - DBA2J mice with streptozotocin induced diabetic nephropathy, I/R model - ischemia/reperfusion model, ADPKD - autosomal dominant polycystic kidney disease, MGN - mesangial glomerulonephritis, FSGS - focal segmental glomerulosclerosis, IGAN - IgA nephropathy, CAN - chronic allograft nephropathy 
sive nephropathy, periostin was expressed predominantly in areas of kidney damage - mainly located around blood vessels. The study showed a significant positive relationship between creatinine concentration, proteinuria, and periostin mRNA and a negative relationship between renal flow and periostin mRNA. In this model, blockage of RAAS with losartan reduced expression of periostin in renal tissue, improved renal hemodynamics, and decreased proteinuria [8]. Similarly, a reduction in RAAS activity was observed after periostin blockade $[65,66]$.

Another pathophysiological pathway proposed is crosstalk between periostin and TGF- $\beta$ signals [46]. This mutual, reciprocal relation has been described in kidneys but also in other human pathologies, e.g., in scleroderma [67]. Periostin promotes adhesion and TGF- $\beta$ release in immune cells. In turn, TGF- $\beta$ induces periostin production in fibroblasts [67]. In kidney tissue, periostin can induce cell dedifferentiation, increase in TGF- $\beta$ expression, and extracellular matrix deposition. TGF- $\beta$ can also promote the expression of periostin, which induces the loss of renal tubular epithelial phenotype (epithelial-mesenchymal transition) and finally leads to renal fibrosis and ESRD [56]. In vitro studies showed that increased periostin expression was accompanied by the loss of E-cadherin, a epithelial cell marker, in tubular cells, which indicates loss of epithelial phenotype [55]. The administration of TGF- $\beta 1$ increased periostin expression and intensified the acquisition of the mesenchymal cell phenotype by tubular cells [56]. Additionally, periostin increased the proliferation of mesangial cells [55]. Prakoura et al. reported that periostin secretion is not always directly activated by TGF- $\beta 1$ and that TGF- $\beta 1$ controls periostin secretion through a Smad independent pathway [68].

The profibrotic role of periostin was also found in human kidney biopsy specimens $[51,52,59]$. In vitro stimulation of renal mesangial cells by external addition of TGF- $\beta 1$ resulted in significant induction of periostin expression and addition of periostin to mesangial cells induced cell proliferation and decreased the number of cells expressing activated caspase-3, a marker of apoptosis [51].

There are many other mechanisms that could explain the role of periostin in kidney pathology. A relationship between periostin and autophagy was revealed. It was shown that periostin blockade decreased the level of pro-inflammatory cytokines and partially restored the level of p62, a well-established negative marker of autophagy. The authors suggest that periostin can activate autophagy by interacting with mTOR kinase complex 1 (mTORC1) [65].

The literature data suggest a relationship between periostin and peroxisome-activated $\alpha$-receptor (PPAR $\alpha$ ). PPAR $\alpha$, a transcription factor regulating fatty acid transport and metabolism, plays a role in cell proliferation and differentiation and exerts anti-inflammatory properties [69, 70]. In the CKD experimental 5/6 nephrectomy model, Bian et al. found that the increase in periostin expression was accompanied by a decrease in PPAR $\alpha$ expression in the injured kidney. Periostin inhibition improved kidney function in rats, increased PPAR $\alpha$ expression, decreased the severity of fibrosis, and improved cardiovascular function in CKD [71]. As PPAR $\alpha$ is able to inhibit TGF- $\beta / \mathrm{Smad} 3$ signaling [72], the authors suggest that the increase in PPAR $\alpha$ activity associated with periostin blockade may reduce ECM deposition due to the TGF- $\beta$ inhibition [71].

There is evidence showing a beneficial role of periostin inhibition in kidney pathology. As already mentioned, periostin blockade with losartan attenuated renal injury in an experimental rat model [8]. Also, Hwang et al. found that periostin blockade was associated with a significant reduction in the areas of fibrosis in the affected kidney. Moreover, periostin-induced significant reductions in the expression of collagen, alpha-smooth muscle actin ( $\alpha$ SMA), and monocyte chemoattractant protein-1 (MCP-1) have been observed [62]. Also, Raman et al. demonstrated that loss of periostin in $p c y / p c y$ mice leads to preservation of kidney function and reduction of fibrosis [73]. In an experimental model of ischemic kidney, An et al. detected a relationship between periostin and p38 mitogen-activated kinase (p38 MAPK). Periostin knockout mice showed lower expression of 338 MAPK than wild-type mice. Administration of p38MAPK inhibitor reduced fibrosis and apoptosis induced by recombinant periostin [74].

In contrast to numerous studies indicating negative effects of periostin, a recent study by Kormann et al. described the protective role of periostin in acute kidney injury (AKI) in a mouse ischemia-reperfusion (I/R) model. Periostin protects against epithelial damage by inhibiting the cell cycle and reducing apoptosis. It was observed that 24 and 72 hours after I/R, mice overexpressing periostin had preserved kidney function, less severe necrosis and dilation of the tubules, and lower expression of proinflammatory cytokines as compared to periostin knockout mice. In addition, in the repair phase, periostin-overexpressing mice showed increased proliferation of macrophages with a pro-regenerative phenotype [75]. Thus, it is possible that the role of periostin in AKI is different from chronic kidney pathologies and resembles the beneficial action of the protein observed in tissue regeneration and wound healing.

\section{Periostin as a potential marker of chronic kidney disease in humans}

In recent years, promising human adult studies have been published that could support the role of periostin as a potential marker of chronic kidney damage and CKD progression.

Sen et al. analyzed the expression of matricellular proteins, e.g., periostin, in the kidney specimens of patients with glomerulopathies. Among all the analyzed proteins, periostin showed the highest expression. The expression of periostin mRNA and the intensity of periostin staining in 
the glomeruli and interstitium correlated negatively with GFR. In patients with preserved renal function periostin expression was limited to glomeruli, whereas in those with renal dysfunction, enhanced periostin expression in the mesangium, tubular interstitium, and sites of fibrosis was found [51]. Also, Satirapoj et al. observed increased glomerular, periglomerular, and tubular expression of periostin in patients with diabetic nephropathy [9] and chronic allograft nephropathy (CAN) [53]. In contrast, periostin was not detected in control kidneys $[9,53]$.

Periostin may play a role as a marker of the progression of kidney disease in lupus nephropathy. Wantanasiri et al. found a significant positive correlation between the chronicity index, segmental and global glomerular sclerosis, interstitial fibrosis, tubular atrophy, and periostin tissue expression. Periostin tissue expression also correlated negatively with GFR. It was found that patients with low disease activity or a low disease chronicity index value but high periostin expression had significantly lower GFR compared to patients with low tissue periostin expression. The authors concluded that periostin kidney expression may be a tissue biomarker associated with the pathogenesis of chronic kidney injury in systemic lupus erythematosus patients [52].

Two studies by Satirapoj et al. analyzed the presence of periostin in the urine of adult patients with CKD. In the first study, the authors found that urinary periostin was significantly higher in CKD subjects compared to the control group. There was no difference between proteinuric and non-proteinuric CKD patients in urinary periostin level. Urinary periostin showed higher sensitivity and specificity in CKD diagnosis compared to neutrophil gelatinase-associated lipocalin (NGAL) [55]. In the second study, the authors analyzed urinary periostin in adult patients with type 2 diabetes mellitus. Urinary periostin levels were significantly elevated in normoalbuminuric, microalbuminuric, and macroalbuminuric patients compared with healthy controls. In diabetic patients, increased urine periostin level correlated significantly with aging, albuminuria, and decline of GFR. The authors concluded that urinary periostin might be used as an early marker of diabetic renal injury [9].

In diabetic patients also serum periostin as a potential biomarker of disease progression was investigated. El-Dawla et al. analyzed serum levels of periostin in patients with type 2 diabetes. The authors found that serum periostin concentration correlated positively with urinary albumin excretion and with concentration of glycated hemoglobin [76].

An et al. analyzed urinary periostin (expressed as periostin/creatinine ratio) in patients with AKI. Urinary periostin at the time of AKI was significantly higher in those who progressed to CKD compared to subjects who did not develop CKD [74].

Periostin concentration in the urine may prove to be an early marker of fibrosis, progression of renal damage, and outcome in IgA nephropathy (IgAN). Hwang et al. reported that patients with high tissue expression of periostin had a significantly higher periostin concentration in the urine. The level of urinary periostin (periostin/creatinine ratio) was significantly higher in patients with interstitial fibrosis/tubular atrophy, interstitial inflammation, hyaline arteriolosclerosis, and glomerular sclerosis in renal biopsy. Urinary periostin correlated negatively with initial GFR and GFR after a mean 27 months of follow-up. High initial urine or tissue periostin levels were significant risk factors for GFR reduction and progression to ESRD [59].

Urinary periostin might be a useful marker of chronic allograft nephropathy (CAN). Satirapoj et al. found that the urinary periostin level was significantly higher in patients with CAN compared to transplant patients with normal renal function and healthy volunteers. In addition, urine periostin levels correlated directly with urine protein creatinine ratio, whereas significant inverse correlations were evidenced with estimated glomerular filtration rate [53].

\section{Summary}

The studies conducted so far show a significant role of periostin in the pathogenesis of kidney diseases. Periostin is involved in inflammation and kidney fibrosis. The exact mechanism of action of periostin in kidney disease has not been established yet, and further studies in the field are warranted. Periostin is highly expressed in various types of kidney diseases (mainly shown in animal models), while its expression in healthy kidneys is not significant. Periostin renal tissue and urinary concentrations increase with the duration and extent of kidney damage and with GFR reduction. The studies performed so far indicate the potential of urinary and tissue periostin as a promising biomarker of CKD progression. As periostin was found to be upregulated in a mouse model of UUO and CAKUT (congenital anomalies of the kidney and urinary tract) is a leading cause of CKD in children, there is a need for studies on the usefulness of periostin as a marker of renal pathology in the pediatric population.

\section{The authors declare no conflict of interest.}

\section{References}

1. Kidney Disease: Improving Global Outcomes (KDIGO) CKD Work Group (2013): KDIGO 2012 Clinical Practice Guideline for the Evaluation and Management of Chronic Kidney Disease. Kidney Int Suppl (2013) 3: 1-150.

2. Kamath N, Iyengar A, George N, et al. (2019): Risk factors and rate of progression of CKD in children. Kidney Int Rep 4: 1472-1477.

3. Harambat J, van Stralen KJ, Kim JJ, et al. (2012): Epidemiology of chronic kidney disease in children. Pediatr Nephrol 27: $363-373$. 
4. Zhou D, Liu Y (2016): Renal fibrosis in 2015: Understanding the mechanisms of kidney fibrosis. Nat Rev Nephrol 12: 68-70.

5. Boor P, Ostendorf T, Floege J (2010): Renal fibrosis: novel insights into mechanisms and therapeutic targets. Nat Rev Nephrol 6: 643-656.

6. Webster AC, Nagler EV, Morton RL, et al. (2017): Chronic kidney disease. Lancet 389: 1238-1252.

7. Theocharis AD, Manou D, Karamanos NK (2019): The extracellular matrix as a multitasking player in disease. FEBS J 286: $2830-2869$.

8. Guerrot D, Dussaule JC, Mael-Ainin M, et al. (2012): Identification of periostin as a critical marker of progression/reversal of hypertensive nephropathy. PLoS One 7: e31974.

9. Satirapoj B, Tassanasorn S, Charoenpitakchai M, et al. (2015): Periostin as a tissue and urinary biomarker of renal injury in type 2 diabetes mellitus. PLoS One 10: e0124055.

10. Takeshita S, Kikuno R, Tezuka K, et al. (1993): Osteoblast-specific factor 2: cloning of a putative bone adhesion protein with homology with the insect protein fasciclin I. Biochem J 294: 271-278.

11. Liu J, Zhang J, Xu F, et al. (2018): Structural characterizations of human periostin dimerization and cysteinylation. FEBS Lett 592: 1789-1803.

12. Hwang EY, Jeong MS, Park EK, et al. (2014): Structural characterization and interaction of periostin and bone morphogenetic protein for regulation of collagen cross-linking. Biochem Biophys Res Commun 449: 425-431.

13. Kudo A (2017): Introductory review: periostin-gene and protein structure. Cell Mol Life Sci 74: 4259-4268.

14. Horiuchi K, Amizuka N, Takeshita S, et al. (1999): Identification and characterization of a novel protein, periostin, with restricted expression to periosteum and periodontal ligament and increased expression by transforming growth factor beta. J Bone Miner Res 14: 1239-1249.

15. Kii I, Ito H (2017): Periostin and its interacting proteins in the construction of extracellular architectures. Cell Mol Life Sci 74: 4269-4277.

16. Kudo A, Kii I (2018): Periostin function in communication with extracellular matrices. J Cell Commun Signal 12: 301308.

17. Norris RA, Damon B, Mironov V, et al. (2007): Periostin regulates collagen fibrillogenesis and the biomechanical properties of connective tissues. J Cell Biochem 101: 695-711.

18. Yun H, Kim EH, Lee CW (2018): $1 \mathrm{H}, 13 \mathrm{C}$, and $15 \mathrm{~N}$ resonance assignments of FAS1-IV domain of human periostin, a component of extracellular matrix proteins. Biomol NMR Assign 12: 95-98.

19. Conway SJ, Izuhara K, Kudo Y, et al. (2014): The role of periostin in tissue remodeling across health and disease. Cell Mol Life Sci 71: 1279-1288.

20. Kii I, Nishiyama T, Li M, et al. (2010): Incorporation of tenascin-C into the extracellular matrix by periostin underlies an extracellular meshwork architecture. J Biol Chem 285: 20282039.

21. González-González L, Alonso J (2018): Periostin: a matricellular protein with multiple functions in cancer development and progression. Front Oncol 8: 225.

22. Olsan EE, West JD, Torres JA, et al. (2018): Identification of targets of IL-13 and STAT6 signaling in polycystic kidney disease. Am J Physiol Renal Physiol 315: F86-F96.

23. Morra L, Moch H (2011): Periostin expression and epithelial-mesenchymal transition in cancer: a review and an update. Virchows Arch 459: 465-475.
24. Morra L, Rechsteiner M, Casagrande S, et al (2012): Characterization of periostin isoform pattern in non-small cell lung cancer. Lung Cancer 76: 183-190.

25. Viloria K, Hill NJ (2016): Embracing the complexity of matricellular proteins: the functional and clinical significance of splice variation. Biomol Concepts 7: 117-132.

26. Kudo A (2019): Periostin in bone biology. Adv Exp Med Biol 1132: 43-47.

27. Oshima A, Tanabe H, Yan T, et al. (2002): A novel mechanism for the regulation of osteoblast differentiation: transcription of periostin, a member of the fasciclin I family, is regulated by the bHLH transcription factor, twist. J Cell Biochem 86: 792-804.

28. Jia YY, Yu Y, Li HJ (2020): The research status and prospect of Periostin in chronic kidney disease. Ren Fail 42: 11661172.

29. Du J, Li M (2017): Functions of periostin in dental tissues and its role in periodontal tissues' regeneration. Cell Mol Life Sci 74: 4279-4286.

30. Cobo T, Obaya A, Cal S, et al. (2015): Immunohistochemical localization of periostin in human gingiva. Eur J Histochem 59: 2548.

31. Bonnet N, Garnero P, Ferrari S (2016): Periostin action in bone. Mol Cell Endocrinol 432: 75-82.

32. Idolazzi L, Ridolo E, Fassio A, et al. (2017): Periostin: The bone and beyond. Eur J Intern Med 38: 12-16.

33. Murota H, Lingli Y, Katayama I (2017): Periostin in the pathogenesis of skin diseases. Cell Mol Life Sci 74: 43214328.

34. Walker JT, McLeod K, Kim S, et al. (2016): Periostin as a multifunctional modulator of the wound healing response. Cell Tissue Res 365: 453-465.

35. Iwayama T, Olson LE (2013): Involvement of PDGF in fibrosis and scleroderma: recent insights from animal models and potential therapeutic opportunities. Curr Rheumatol Rep 15: 304.

36. Yamaguchi Y, Ono J, Masuoka M, et al. (2013): Serum periostin levels are correlated with progressive skin sclerosis in patients with systemic sclerosis. Br J Dermatol 168: 717-725.

37. Adam O, Löhfelm B, Thum T, et al. (2012): Role of miR-21 in the pathogenesis of atrial fibrosis. Basic Res Cardiol 107: 278.

38. Snider P, Hinton RB, Moreno-Rodriguez RA, et al. (2008): Periostin is required for maturation and extracellular matrix stabilization of noncardiomyocyte lineages of the heart. Circ Res 102: 752-760.

39. Landry NM, Cohen S, Dixon IMC (2017): Periostin in cardiovascular disease and development: a tale of two distinct roles. Basic Res Cardiol 113: 1.

40. Horne TE, VandeKopple M, Sauls K, et al. (2015): Dynamic heterogeneity of the heart valve interstitial cell population in mitral valve health and disease. J Cardiovasc Dev Dis 2: 214-232.

41. Wang D, Oparil S, Feng JA, et al. (2003): Effects of pressure overload on extracellular matrix expression in the heart of the atrial natriuretic peptide-null mouse. Hypertension 42: 88-95.

42. Oka T, Xu J, Kaiser RA, et al. (2007): Genetic manipulation of periostin expression reveals a role in cardiac hypertrophy and ventricular remodeling. Circ Res 101: 313-321.

43. O'Dwyer DN, Moore BB (2017): The role of periostin in lung fibrosis and airway remodeling. Cell Mol Life Sci 74: 43054314.

44. Izuhara K, Ohta S, Ono J (2016): Using periostin as a biomarker in the treatment of asthma. Allergy Asthma Immunol Res 8: 491-498. 
45. Ashley SL, Wilke CA, Kim KK, et al. (2017): Periostin regulates fibrocyte function to promote myofibroblast differentiation and lung fibrosis. Mucosal Immunol 10: 341-351.

46. Nanri Y, Nunomura S, Terasaki Y, et al. (2020): Cross-talk between transforming growth factor- $\beta$ and periostin can be targeted for pulmonary fibrosis. Am J Respir Cell Mol Biol 62: 204-216.

47. Maxfield AZ, Landegger LD, Brook CD, et al. (2018): Periostin as a biomarker for nasal polyps in chronic rhinosinusitis. Otolaryngol Head Neck Surg 158: 181-186.

48. Okazaki T, Tamai K, Shibuya R, et al. (2018): Periostin is a negative prognostic factor and promotes cancer cell proliferation in non-small cell lung cancer. Oncotarget 9: 31187 31199.

49. Ratajczak-Wielgomas K, Grzegrzolka J, Piotrowska A, et al. (2017): Expression of periostin in breast cancer cells. Int J Oncol 51: 1300-1310.

50. Morra L, Rechsteiner M, Casagrande S, et al. (2011): Relevance of periostin splice variants in renal cell carcinoma. Am J Pathol 179: 1513-1521.

51. Sen K, Lindenmeyer MT, Gaspert A, et al. (2011): Periostin is induced in glomerular injury and expressed de novo in interstitial renal fibrosis. Am J Pathol 179: 1756-1767.

52. Wantanasiri P, Satirapoj B, Charoenpitakchai M, et al. (2015): Periostin: a novel tissue biomarker correlates with chronicity index and renal function in lupus nephritis patients. Lupus 24: 835-845.

53. Satirapoj B, Witoon R, Ruangkanchanasetr P, et al. (2014): Urine periostin as a biomarker of renal injury in chronic allograft nephropathy. Transplant Proc 46: 135-140.

54. Sorocos K, Kostoulias X, Cullen-McEwen L, et al. (2011): Expression patterns and roles of periostin during kidney and ureter development. J Urol 186: 1537-1544.

55. Satirapoj B, Wang Y, Chamberlin MP, et al. (2012): Periostin: novel tissue and urinary biomarker of progressive renal injury induces a coordinated mesenchymal phenotype in tubular cells. Nephrol Dial Transplant 27: 2702-2711.

56. Mael-Ainin M, Abed A, Conway SJ, et al. (2014): Inhibition of periostin expression protects against the development of renal inflammation and fibrosis. J Am Soc Nephrol 25: 17241736.

57. Wallace DP, Quante MT, Reif GA, et al. (2008): Periostin induces proliferation of human autosomal dominant polycystic kidney cells through alphaV-integrin receptor. Am J Physiol Renal Physiol 295: F1463-1471.

58. Haertel-Wiesmann M, Liang Y, Fantl WJ, et al. (2000): Regulation of cyclooxygenase- 2 and periostin by Wnt-3 in mouse mammary epithelial cells. J Biol Chem 275: 32046-32051.

59. Hwang JH, Lee JP, Kim CT, et al. (2016): Urinary periostin excretion predicts renal outcome in IgA nephropathy. Am J Nephrol 44: 481-492.

60. Maruhashi T, Kii I, Saito M, Kudo A (2010): Interaction between periostin and BMP-1 promotes proteolytic activation of lysyl oxidase. J Biol Chem 285: 13294-13303.

61. Kang YS, Li Y, Dai C, et al. (2010): Inhibition of integrin-linked kinase blocks podocyte epithelial-mesenchymal transition and ameliorates proteinuria. Kidney Int 78: 363 373.

62. Hwang JH, Yang SH, Kim YC, et al. Experimental inhibition of periostin attenuates kidney fibrosis. Am J Nephrol 46: 501-517.

63. Li L, Fan D, Wang C, et al. (2011): Angiotensin II increases periostin expression via Ras/p38 MAPK/CREB and ERK1/2/
TGF- $\beta 1$ pathways in cardiac fibroblasts. Cardiovasc Res 91: 80-89.

64. Wu H, Chen L, Xie J, et al. (2016): Periostin expression induced by oxidative stress contributes to myocardial fibrosis in a rat model of high salt-induced hypertension. Mol Med Rep 14: 776-782.

65. Bian X, Bai Y, Su X, et al. (2019): Knockdown of periostin attenuates 5/6 nephrectomy-induced intrarenal renin-angiotensin system activation, fibrosis, and inflammation in rats. J Cell Physiol 234: 22857-22873.

66. Ishigaki S, Ohashi N, Matsuyama T, et al. (2018): Melatonin ameliorates intrarenal renin-angiotensin system in a $5 / 6$ nephrectomy rat model. Clin Exp Nephrol 22: 539-549.

67. Izuhara K, Nunomura S, Nanri Y, et al. (2017): Periostin in inflammation and allergy. Cell Mol Life Sci 74: 4293-4303.

68. Prakoura N, Kavvadas P, Kormann R, et al. (2017): NFkB-induced periostin activates integrin- $\beta 3$ signaling to promote renal injury in GN. J Am Soc Nephrol 28: 1475-1490.

69. Berger J, Moller DE (2002): The mechanisms of action of PPARs. Annu Rev Med 53: 409-435.

70. Cheng HS, Tan WR, Low ZS (2019): Exploration and development of PPAR modulators in health and disease: an update of clinical evidence. Int J Mol Sci 20: 5055.

71. Bian X, Su X, Wang Y, et al. (2019): Periostin contributes to renal and cardiac dysfunction in rats with chronic kidney disease: Reduction of PPAR $\alpha$. Biochimie 160: 172-182.

72. Lyu H, Li X, Wu Q, Hao L (2019): Overexpression of microRNA-21 mediates Ang II-induced renal fibrosis by activating the TGF- $\beta 1 / \mathrm{Smad} 3$ pathway via suppressing PPAR $\alpha$. J Pharmacol Sci 141: 70-78.

73. Raman A, Parnell SC, Zhang Y, et al. (2018): Periostin overexpression in collecting ducts accelerates renal cyst growth and fibrosis in polycystic kidney disease. Am J Physiol Renal Physiol 315: F1695-F1707.

74. An JN, Yang SH, Kim YC, et al. (2019): Periostin induces kidney fibrosis after acute kidney injury via the p38 MAPK pathway. Am J Physiol Renal Physiol 316: F426-F437.

75. Kormann R, Kavvadas P, Placier S, et al. (2020): Periostin promotes cell proliferation and macrophage polarization to drive repair after AKI. J Am Soc Nephrol 31: 85-100.

76. El-Dawla NMQ, Sallam AM, El-Hefnawy MH, et al. (2019): E-cadherin and periostin in early detection and progression of diabetic nephropathy: epithelial-to-mesenchymal transition. Clin Exp Nephrol 23: 1050-1057.

77. Zhao X, Hao J, Duan H, et al. (2017): Phosphoinositide 3-kinase/protein kinase $\mathrm{B} /$ periostin mediated platelet-derived growth factor-induced cell proliferation and extracellular matrix production in lupus nephritis. Exp Biol Med (Maywood) 242: $160-168$

78. Alfieri C, Regalia A, Moroni G, et al. (2019): Novel markers of graft outcome in a cohort of kidney transplanted patients: a cohort observational study. J Nephrol 32: 139-150. 